\title{
Manejo de sulfatação nas crises hipertensivas gestacionais
}

\author{
Management of sulfatation in gendering hypertensive crises \\ Manejo de sulfatación en las crisis hipertensivas gestacionales
}

\begin{abstract}
Kleberson Adriano Silva Gomes ${ }^{1 *}$, Stefano dos Santos Adorno1, Maria Luiza Pinto Ramalho', Mário Clodoaldo Batista da Costa1, Moisés Castro Saback¹.
\end{abstract}

\section{RESUMO}

Objetivo: Esclarecer sobre as possibilidades de métodos de sulfatação em meio a crises hipertensivas graves gestacionais, e seus benefícios de tal prática específica. Métodos: Foi realizado o método de pesquisa descritivo por intermédio de revisão bibliográfica básica e sistemática, desenvolvida com a seleção de artigos em base de dados de pesquisas em saúde, utilizando-se dos seguintes descritores: sulfatação, pré-eclâmpsia, gestantes, benefícios. Resultados: Observou-se que os resultados alcançados, com base nos critérios de seleção dos artigos/publicações acadêmicas, foram desenvolvidos direcionando aos interesses buscados nesta pesquisa. Conclusão: A respeito à padronização de um modelo de sulfatação a ser seguido, é apropriado afirmar que mesmo existindo provas dos benefícios propostos pelo uso correto da medicação quando padronizado, esbarramos nas variâncias de um modelo padrão em diversos serviços obstétricos.

Palavras-chave: Crise hipertensiva gestacional, Pré-eclâmpsia, Gestação, Relação materno-fetal.

\begin{abstract}
Objective: To clarify the possibilities of sulphation methods in the context of severe gestational hypertensive crises, and their benefits from such specific practice. Methods: The descriptive research method was carried out through a basic and systematic bibliographic review, developed with the selection of articles in a health research database, using the following descriptors: sulfation, preeclampsia, pregnant women, benefits. Results: It was observed that the results obtained, based on the selection criteria of the articles / academic publications, were developed directing the interests sought in this research. Conclusion: regarding the standardization of a model of sulfation to be followed, it is appropriate to state that even if there is evidence of the benefits proposed by the correct use of the medication when standardized, we come across the variances of a standard model in several obstetric services.
\end{abstract}

Keywords: Gestational hypertensive crisis, Pre-eclampsia, Gestation, Mother-fetal relationship.

\section{RESUMEN}

Objetivo: Esclarecer sobre las posibilidades de métodos de sulfatación en medio de crisis hipertensivas graves gestacionales, y sus beneficios de tal práctica específica. Métodos: Se realizó el método de investigación descriptivo por medio de una revisión bibliográfica básica y sistemática, desarrollada con la selección de artículos en base de datos de investigaciones en salud, utilizando los siguientes descriptores: sulfatación, preeclampsia, gestantes, beneficios. Resultados: Se observó que los resultados alcanzados, sobre la base de los criterios de selección de los artículos / publicaciones académicas, fueron desarrollados dirigiendo a los intereses buscados en esta investigación. Conclusión: respecto a la estandarización de un modelo de sulfatación a seguir, es apropiado afirmar que incluso existiendo pruebas los beneficios propuestos por el uso correcto de la medicación cuando estandarizado, chocamos en las varianzas de un modelo estándar en diversos servicios obstétricos.

Palabras clave: Crisis hipertensiva gestacional, Pre-eclampsia, Gestación, Relación materno-fetal.

1 Universidade Estadual do Amazonas (UEA), Manaus - AM. *E-mail: klegomes@hotmail.com 


\section{INTRODUÇÃO}

As síndromes hipertensivas na gestação merecem especial destaque no cenário da saúde pública mundial. Atualmente, representam a terceira causa de mortalidade materna no mundo, perdendo apenas para hemorragia pós-parto e infecção pós-parto, respectivamente. No Brasil é a primeira causa. Em países desenvolvidos, aproximadamente de duas a oito em cada 100 gestantes vão desenvolver o evento enquanto no Brasil, pode-se chegar a 10\% dos casos. Devido à gravidade da doença, é considerada como causa de internamento em unidade de terapia intensiva e, por vezes, incluída como critério de morbidade materna grave (ZUGAIB, 2008).

Segundo Silva (2011), entende-se, por meio de inúmeros relatos de casos e estudos prospectivos relacionados a crises hipertensivas na gestação, que a utilização conjunta de certos hipotensores orais como, por exemplo, a nifedipina junto ao sulfato de magnésio $(\mathrm{Mg})$ venoso pode potencializar o bloqueio neuromuscular, bem como promover vasodilatação arterial com desfecho anti-hipertensivo e anticonvulsivante nas situações de crises em gestantes

A condição de eclâmpsia consiste na manifestação convulsiva de estado grave da pré-eclâmpsia (PE), desenvolvendo-se de maneira isolada nesse estado clínico ou ainda presente nas gestações com hipertensão arterial já preexistente (SILVA et. al, 2013).

Silva (2011),descreve, que a eclâmpsia é uma séria complicação da gravidez, além de ser caracterizada pelo acometimento de convulsões nas pacientes, é também um acidente agudo paroxístico da toxemia gravídica, sendo a forma mais grave da PE, que consiste em acessos convulsivos persistentes podendo gerar eventos catastróficos seguidos algumas vezes por um estado comatoso.

Diretamente associada com a comorbidade está a PE, quadro que se apresentam nas formas leve, moderada ou grave. É uma doença caracteristicamente multissistêmica, que ocorre especialmente após a $20^{a}$ semana de gestação e mostra determinadas manifestações clínicas características da patologia: hipertensão, edema e proteinúria (presença de proteínas na urina). Nas formas graves, a PE surge com acometimentos clínicos severos na gestante, dentre elas a irritabilidade de todo o sistema nervoso central, alterações visuais e gastrointestinais, podendo evoluir com convulsões, de maneira que a doença passa a ser denominada eclâmpsia; por outro lado, se ausente à crise convulsiva, caracteriza-se nas três formas já citadas previamente (REZENDE; MONTENEGRO, 2006).

Segundo Amorim; Souza (2009), as síndromes hipertensivas são representação de uma das alterações que ocorrem com mais frequência no decorrer da gravidez, e que se encontram entre as principais causas de morte materno-fetal e perinatal em todo o mundo.

Por isso, entende-se que o processo de identificação de pacientes de risco com crises hipertensivas, com potencial para o desenvolvimento da PE gestacional, dá-se em função de fatores associados ou por testes preditores, seguido de pontuais intervenções profiláticas que poderiam prevenir ou retardar a apresentação clínica da doença ou, até mesmo, chegar a reduzir sua gravidade (AMORIM e SOUZA, 2009).

Bezerra et al (2005) afirma que a patologia analisada, além de ser a maior responsável pela morbidade/mortalidade materna e fetal, também é responsabilizada pelo grande número de mortes perinatais, índices elevados de prematuridade e a ocorrência de retardo de crescimento intrauterino.

Em continuidade, Silva (2011), evidencia que a depender da gravidade e da evolução clínica da paciente ou ainda em casos de sofrimento fetal agudo, torna-se autorizada à interrupção da gestação, quando a idade gestacional exceder a 34 semanas.

Mediante a todo cenário negativo imposto pela doença, conta-se com uso do sulfato de MG como a principal medicação no tratamento das crises convulsivas gestacionais, propedêutica descrita primeiramente por Lazard em 1925 e, posteriormente, passando a ser reconhecido e recomendado por diversos centros obstétricos também durante a prevenção da crise convulsiva gestacional, diante a PE grave com apresentação dos sinais de iminência de eclâmpsia $O$ risco de iminência de eclâmpsia e sua associação com 
aumento da morbidade e mortalidade materna e perinatal, principalmente nos países em desenvolvimento, justifica a larga utilização da terapia anticonvulsivante na prevenção de crises convulsivas em gestantes com PE.(SOUZA, 2009).

Segundo Prado et al (1991) as pacientes com eclâmpsia devem ser sulfatadas imediatamente, em meio ao quadro de convulsão gestacional, ou ainda antes, especificamente em casos de PE grave associado a sinais iminência de eclâmpsia. E os principais esquemas de sulfatação, que atualmente são utilizados seriam os esquemas de Pritchard, Zuspan e Sibai.

\section{MÉTODOS}

A pesquisa se faz por meio de revisão bibliográfica de caráter sistemático, utilizando como fonte de dados à literatura científica existente sobre os métodos disponíveis no tratamento das crises hipertensivas gestacional. As fontes para a localização das publicações foram bases de dados eletrônicas: Periódicos da Biblioteca Virtual em Saúde (BVS); Coordenação de Aperfeiçoamento de Pessoal de Nível Superior (CAPES); Scientific Eletronic Library online (SCIELO) e Literatura Latino-americana e do Caribe em Ciências da Saúde (LILACS). A busca dos dados foi realizada de acordo com os seguintes descritores: métodos de sulfatação, crise hipertensiva gestacional, eclâmpsia, gestação, relação materno-fetal e benefícios. Utilizando os pressupostos contidos em meio aos critérios, de inclusão e exclusão.

\section{RESULTADOS E DISCUSSÃO}

Zuspan (1966) descreveu um dos mais utilizados esquemas de administração do sulfato de magnésio, o endovenoso, no qual é administrada uma dose inicial de $4 \mathrm{~g}$ de sulfato de magnésio e uma dose contínua de manutenção de $1 \mathrm{~g}$ por hora, ambas aplicadas por via intravenosa, que deve ser mantido por pelo menos 24 horas (BATISTA, 2015).

A dose de manutenção de sulfato de magnésio do esquema de Zuspan não coloca-se como sendo suficiente para o atingimento e manutenção de concentrações terapêuticas e, assim, evidenciou a proposição de aumentar a concentração da dose de manutenção para $2 \mathrm{~g}$ por hora, em infusão contínua, por ao menos 24 horas, intencionando especificamente atingir as mesmas concentrações séricas proposta pelo tratamento por via intramuscular dimensionado por Pritchard (BATISTA, 2015).

Neste interim, os autores Bhattacharjee et al. (2011), expõe que, o esquema proposto por Pritchard (1955) a dose inicial é de $10 \mathrm{~g}$ de sulfato de magnésio por via intramuscular em conjunto com $4 \mathrm{~g}$ infundida por via intravenosa em "bolus", destarte, a dose de manutenção é de $5 \mathrm{~g}$ via intramuscular a ser aplicada a cada 4 horas possuem inicialmente níveis de concentração terapêutica adequado para controle convulsivo nas gestantes.

Batista (2015), ainda depreende que o modelo de Pritchard traz determinações ao fato de que, a concentração sérica do íon magnésio, para proporcionar prevenção ou tratamento da condição de eclâmpsia, deve ser categoricamente maior do que a concentração sérica normal, sendo importante o esclarecimento de que esta concentração deve alcançar variação entre 4,8 e $8,4 \mathrm{mg} / \mathrm{dL}$, o que de fato ocorre durante aplicação da dose de ataque neste esquema.

Existem diferenciações dos modelos propostos na literatura, por exemplo, Maia et al. (2014), promoveram comparações acerca do uso de sulfato de magnésio por 12 horas e por 24 horas em mulheres no pós-parto associada com pré-eclâmpsia grave já estável. Como resultados, os autores demonstraram que a terapia de sulfato de magnésio abreviado (desenvolvida por 12 horas) associa-se a um menor tempo de exposição ao sulfato de magnésio e, clinicamente, em ambos os grupos foram encontradas reduções significativas na duração do uso no pós-parto de um cateter de bexiga permanente (em pacientes com insuficiência renal), controle da pressão arterial antes, durante e após o parto, diminuição do tempo de hospitalização e redução do tempo para efetivamente acontecer o contato materno para com o recém-nascido.

REAS/EJCH | Vol.Sup.19 | e222 | DOI: https://doi.org/10.25248/reas.e222.2019 Página 3 de 6 
Por outro lado, os autores Charoenvidhya; Manotaya (2013), em estudo randomizado com dois grupos de pacientes utilizaram-se uma dose inicial de sulfatação com a concentração de $5 \mathrm{~g}$ de sulfato de magnésio administrada por via intravenosa associado com doses de manutenção de $2 \mathrm{~g} / \mathrm{hora}$ e $1 \mathrm{~g} / \mathrm{hora}$ contínuas por até 24 horas do pós-parto, conclusivamente, demonstrou-se que a dose de manutenção de sulfato de magnésio mais eficaz e mais provável para o alcance do nível terapêutico de magnésio foi a de $2 \mathrm{~g} / \mathrm{hora}$.

Silva (2011) relata os benefícios do manejo da sulfatação com dose de manutenção de $2 \mathrm{~g} / \mathrm{hora}$ por via intravenosa, mas ressalva indispensável monitorar as concentrações séricas de $\mathrm{Mg}$, observando ainda a diurese e a hidratação da paciente para analisar efeitos positivos e negativos de tal uso.

Mundle et al. (2012), esclarecem a importância da utilização da bomba de infusão contínua, oferecendo uma alternativa à administração intramuscular de sulfato de magnésio (tal como propõe Pritchard), levando a menos náuseas, dor de cabeça, risco de hematomas e abscesso derivado da aplicação e dor em geral, além de diminuir efeitos colaterais e de circunstâncias como toxicidade, oligúria (diminuição da produção de urina) ou de insuficiência renal.

Para Bhattacharjee et al. (2011), depreende-se que o uso de sulfato de magnésio intravenoso mesmo em baixa dose (low-dose) foi vista como tão eficaz quanto a viabilização do regime intramuscular padrão proposto por Pritchard, devendo ser observada a manutenção de uma margem de segurança elevada.

Neste ínterim, os autores Mahajan et al. (2009), depreendem ainda que o regime de baixa dose de sulfatação parece proporcionar controle e prevenção a ocorrência de convulsões de forma efetiva em mulheres indianas, de forma que, durante o estudo prospectivo por eles realizado todas as mulheres participantes não apresentaram sinais de alerta quando submetidas a droga.

Em conformidade com os esclarecimentos propostos pelo estudo de Lotufo (2016), compreende-se que as possíveis manifestações de toxicidade associadas com o uso da sulfatação por magnésio estão, não apenas relacionadas com a dose aplicada, também com o tempo de infusão a que a mãe tiver sido submetida.

Geralmente, infusões por mais de 24 horas (todos os protocolos elucidados na literatura especializada têm duração de até 24 horas), podem levar ao desenvolvimento frequente de manifestações clínicas para o recémnascido. Porém, o autor releva importância ao fato que médicos de variados serviços de saúde, recorrentemente, não prescrevem o medicamento de forma adequada justificado em função de sua toxidade (LOTUFO, 2016).

Mesmo com os benefícios da sulfatação de magnésio, os autores Aluma et al. (2014), enfatiza que os níveis de sulfato de magnésio nas concentrações séricas de mulheres grávidas com crises hipertensivas devem ser rigorosamente controlados, porém, não define valores em relação a dose toxicidade para o uso de tal substância.

Em casos singulares, poderia haver uma contraindicação relativa para administração do sulfato de magnésio em pacientes com insuficiência renal aguda e insuficiência renal crônica, já que a via de excreção é através das funções renais. Mas, a terapia pode ser controlada levando em consideração as mensurações em série do magnésio plasmático com exames laboratoriais, junto ao controle clínico rigoroso, aplicando-se a metade da dose do medicamento em concordância com nível de creatinina sérica inferior a 1,5 mg/dL da paciente, reiterando os benefícios do sulfato mesmo em baixas doses (ALUMA et al., 2014).

Complementando entendimentos, retoma-se o esclarecimento de que a intoxicação por sulfato de magnésio, que costuma ser geralmente rara, pode ser identificada através da avaliação clínica, observandose fatores como diurese materna, frequência respiratória e reflexos patelares, sem necessidade de ser dosado a magnesemia, o que é de grande valia em serviços de saúde desprovidos de laboratório para tal finalidade, e na eventualidade de intoxicação por sulfato de magnésio o quadro é revertido por seu antídoto, o gluconato de cálcio (RUANO et al., 2014).

Em documento não oficial, uma bula do sulfato de magnésio, foi encontrado dimensionamento sobre a superdose do medicamento, com a qual pode ser percebido o acometimento de náuseas, calores, sonolência, 
dupla visão, fala inarticulada e fraqueza, sintomas estes que se desenvolvem quando a concentração do sulfato de magnésio nos níveis plasmáticos for entre 9 a $12 \mathrm{mg} / \mathrm{dL}$, seguindo de paralisia muscular e determinadas dificuldades respiratórias podem ser observadas quando dos níveis plasmáticos de 15 a 17 $\mathrm{mg} / \mathrm{dL}$ (TOPHARMA, 2010).

Desta feita, a intoxicação por sulfato de magnésio geralmente apresenta manifestação quando há queda acentuada da diurese, dificuldade respiratória decorrente de paralisia muscular e desaparecimento de reflexos patelares. Se a função renal da paciente for caracterizada como deficiente, pode ser necessária a realização de diálise para a eliminação do sulfato de magnésio da corrente sanguínea (TOPHARMA, 2010).

O sulfato de magnésio mantém-se absoluto como anticonvulsivante eleito para aplicação em mulheres com pré-eclâmpsia grave ou eclâmpsia, devendo ser administrado seguindo os protocolos vigentes em um regime completo de sulfatação no pré-parto, intraparto e pós-parto. Se o processo de administração de um regime completo não for efetivamente possível, as pacientes deverão receber uma dose de ataque de sulfato de magnésio e, posteriormente, serem transferidas de imediato para uma unidade de cuidados intensivos de saúde para a promoção de tratamento adicional e, por fim, completo (FREITAS, 2014, CUESTA, 2016).

Resultados satisfatórios da sulfatação com magnésio referem-se, principalmente, à diminuição do índice de morbimortalidade da gestante com recorrentes crises hipertensivas, do feto e, também, do recém-nascido, possibilitando à mulher uma oportunidade de seguir uma gravidez tranquila e sem qualquer tipo de intercorrências (ANJOS et al., 2014).

Além disso, o uso de sulfato de magnésio em pacientes pré-eclâmpticas ou eclâmpticas reflete resultados positivos no papel neuroprotetor fetal, quando realizado com eficácia e precisão em mulheres que margeiam o risco de parto prematuro. Promove efeito direto sobre o desenvolvimento da função motora na primeira infância e na infância tardia relaciona-se com desenvolvimento geral e cognitivo da criança (DULEY et al., 2010).

Ainda sobre benefícios fetais, o sulfato de magnésio possui efeitos positivos em relação à proteção neurológica fetal, especialmente, quando do nascimento em idades gestacionais precoces (prematuros menor de 32 semanas), porém, para o alcance de uma melhor definição e elegibilidade do uso de sulfatação direcionado para a neuroproteção fetal, outros estudos randomizados e de controle devem ser realizados com maior constância (MORON et al., 2013).

\section{CONCLUSÃO}

Com a percepção adquirida neste estudo, é firmado que o dimensionamento da realidade assistencial da população gravídica em todas as regiões, acometidas pela doença hipertensiva gestacional grave, é totalmente carente de profissionais especializados, padronização no manejo do sulfato de magnésio, estrutura hospitalar de emergência adequada, déficit de materiais de trabalho e até mesmo falta de medicação. Estes são subsídios que embasam a má condução das gestantes englobadas no grupo de risco, e mesmo existindo protocolos já estabelecidos de sulfatação para gestantes, isto não quer dizer que eles serão aplicados corretamente, sendo assim, reitera-se a necessidade de promover fortemente a adesão com a padronização de um esquema de sulfatação com magnésio nas grávidas acometidas pela pré-eclâmpsia graves e / ou eclâmpsia. Estes obedecendo aos métodos descritos, onde, esquemas consolidam o potencial de sobrevida a este grupo de risco, tanto para as gestantes como para os fetos intra-útero e recém-nascidos. 


\section{REFERÊNCIAS}

1. ALUMA RV et al. Intoxicación por sulfato de magnesio en pacientes con preeclampsia y eclampsia e insuficiencia renal. Rev Chil Obstet Ginecol 2014;69(1):44-47.

2. AMORIM MMR, SOUZA ASR. Prevenção da pré-eclâmpsia baseada em evidências. FEMINA, jan. 2009;37(1):47-52.

3. ANJOS JCS, et al. Perfil epidemiológico das gestantes atendidas em um centro de referência em pré-natal de alto risco. Revista Paraense de Medicina, abr./jun. 2014;28(2):23-33.

4. BATISTA FRG. Concentração sérica de sulfato de magnésio em gestantes com pré-eclâmpsia, submetidas aos esquemas de Zuspan e de Sibai. Dissertação (Mestrado). Universidade Estadual Paulista Júlio de Mesquita Filho. Botucatu: Faculdade de Medicina de Botucatu, 2015.

5. BEZERRA EHM, et al. Mortalidade materna por hipertensão: índice e análise de suas características em uma maternidadeescola. Rev Bras Ginecol Obstet, 2005;27(9):548-553

6. BHATTACHARJEE N, et al. A randomized comparative study between low-dose intravenous magnesium sulphate and standard intramuscular regimen for treatment of eclampsia. J Obstet Gynaecol. 2011;31(4):298-303.

7. CHAROENVIDHYA D, MANOTAYA S. Magnesium sulfate maintenance infusion in women with preeclampsia: a randomized comparison between 2 gram per hour and 1 gram per hour. J Med Assoc Thai. Apr. 2013;96(4):395-398.

8. CUESTA YD. Estratégias para assistência à gestante com hipertensão arterial sistêmica, equipe de saúde da família Cidade Nova, Santana do Paraíso/MG. Trabalho de Conclusão de Curso (Especialização). Curso de Especialização Estratégia Saúde da Família. Ipatinga: Universidade Federal de Minas Gerais, 2016

9. DULEY L, et al. Magnesium sulphate and other anticonvulsants for women with pre-eclâmpsia. Cochrane Database Syst Rev. 2010;(12):CD000025.

10. FREITAS D R. Avaliação do estado nutricional e seus determinantes em gestantes atendidas em uma maternidade pública da cidade de Manaus/AM - 2011. Dissertação (Mestrado). Mestrado em Ciências de Alimentos. Manaus: Universidade Federal do Amazonas. Faculdade de Ciências Farmacêuticas, 2014.

11. LAZARD E M. A preliminary report on the intravenous use of magnesium sulphate in puerperal eclâmpsia. Am J Obstet Gynecol. 1925;9(2):178-88.

12. LOTUFO F A H. Acesso ao sulfato de magnésio na pré-eclâmpsia grave e eclâmpsia: desafios organizacionais e profissionais no sistema público de saúde. Tese (Doutorado). Programa de Pós-Graduação em Tocoginecologia. Faculdade de Ciências Médicas. Campinas: Universidade Estadual de Campinas, 2016.

13. MAHAJAN N. et al. "Padhar regime" - a low-dose magnesium sulphate treatment for eclampsia. Gynecol Obstet Invest. 2009;67:20-24.

14. MAIA SB, Katz et al.Abbreviated (12-hour) versus traditional (24-hour) postpartum magnesium sulfate therapy in severe preeclampsia. Int J Gynaecol Obstet. Sep. 2014;126(3):260-264.MORON et al., 2013).

15. MUNDLE S, et al. Treatment approaches for preeclampsia in low-resource settings: A randomized trial of the Springfusor pump for delivery of magnesium sulfate. Int J Womens Cardiovascular Health Preg Hyperten. 2012;2(1):32-38.

16. PRADO FC, et al. Atualização Terapêutica, Manual Prático de Diagnóstico e Tratamento. 15 ed. Rio de Janeiro: Artes Médicas, 1991.

17. REZENDE J, MONTENEGRO, A C B. Obstetrícia fundamental. 10 ed. Rio de Janeiro: Guanabara Koogan; 2006.

18. RUANO R, et al. Sulfato de magnésio (MgSO4) no tratamento e prevenção da eclâmpsia: qual esquema adotar? Rev. Assoc. Med. Bras., São Paulo, jul./set. 2014;50(3).

19. SILVA V, et al TGB. Sulfatação na eclâmpsia - revisão de literatura. Revista UNINGÁ Review, out./dez. 2013;16(1):55-58.

20. SILVA, YCC. Monitoramento emergencial de gestante portadora de Doença Hipertensiva Específica da Gravidez (DHEG) relato de caso clínico. Monografia (Especialização). Programa de Pós-Graduação em enfermagem em emergências. Salvador: Universidade Castelo Branco, 2011.

21. SOUZA, R G. Hipertensão arterial na gestação: avaliação dos resultados maternos e perinatais na Maternidade Carmela Dutra de 2002 a 2009. Trabalho de Conclusão de Curso (Graduação). Florianópolis: Universidade Federal de Santa Catarina. Curso de Graduação em Medicina, 2009.TOPHARMA, 2010 